\title{
Laparoscopic treatment of cholelithiasis in cirrhotic patients
}

\author{
M. Flores Cortés, A. Obispo Entrenas, F. Docobo Durántez, E. Romero Vargas, D. Legupín Tubío and \\ Z. Valera García
}

Department of General and Gastrointestinal Surgery. University Hospital Virgen del Rocío. Seville, Spain

\begin{abstract}
Objective: to assess the safety and efficacy of laparoscopy in the treatment of symptomatic cholelithiasis in patients with Child's Class A and Class B cirrhosis.

Study design: descriptive and retrospective study.

Patients: we studied 14 patients (mean age 60 yrs) with Child's Class A and Class B hepatic cirrhosis who underwent laparoscopic cholecystectomy. We analyzed the occurrence of intraoperative and postoperative complications.

Results: eight patients were women (57.14\%) and 6 were men (42.85\%). Eight of the 14 patients presented with Child's Class B cirrhosis and 6 patients with Class A. Cholecystectomy was programmed for all patients. The average duration of surgery was 77 min. Intraoperative complications occurred in 2 patients (14.28\%) in the form of liver bed bleeding. Postoperative complications were observed in 3 patients $(21.42 \%), 2$ presented with ascites which led to a worsening of Child's Class in one of them, and the third patient presented with angina-like symptoms (acute, sharp pain in the chest irradiating to the back). Mean length of hospital stay was 3 days. No postoperative morbidity or mortality occurred, and there were no conversions.

Conclusions: LC (laparoscopic cholecystectomy) is a safe and effective alternative for the treatment of symptomatic cholelithiasis in patients with well-compensated Child's Class A and Class B cirrhosis. Postoperative morbi-mortality is low, bleeding is unimportant, and both duration of surgical procedure and hospital stay are short.
\end{abstract}

Key words: Cirrhosis. Laparoscopic cholecystectomy. Cholecystectomy.

Flores Cortés M, Obispo Entrenas A, Docobo Durántez F, Romero Vargas E, Legupín Tubío D, Valera García Z. Laparoscopic treatment of cholelithiasis in cirrhotic patients. Rev Esp Enferm Dig 2005; 97: 648-653.

Recibido: 27-10-04.

Aceptado: 10-05-05.

Correspondencia: Mercedes Flores Cortés. C/ Cornisa Azul, edif. 3 n 2 4E. 41290 San Juan de Aznalfarache. Sevilla.

\begin{abstract}
ABBREVIATIONS
LC: laparoscopic cholecystectomy; OC: open cholecystectomy; ASA: American Society of Anesthesiologists.
\end{abstract}

\section{INTRODUCTION}

Since Mouret and Dubois first published in 1988 their experience with laparoscopic cholecystectomy (LC), laparoscopy has become the procedure of choice to treat symptomatic cholelithiasis due to its low invasiveness, reduced postoperative discomfort, shorter hospital stay, and especially safety and efficacy.

Nevertheless, the indication of laparoscopy in cirrhotic patients remains a controversial issue since it may increase the risk of perioperative morbimortality due to lack of experience in using laparoscopy for this type of patients.

On the other hand, only a few thorough publications have evaluated the benefits and safety of this procedure in cirrhotic patients so far (R). Most of the reports published over the last 15 years advocate for the use of laparoscopy as an alternative to laparotomy in the early stages of cirrhosis.

The aim of the present study was to assess the prevalence of LC in the treatment of symptomatic cholelithiasis in cirrhotic patients, as well as its safety and efficacy to cure this disease.

\section{MATERIAL AND METHODS}

We conducted a retrospective, descriptive study of all LCs performed in our hospital in cirrhotic patients from January 1996 through November 2003. During this period, 14 cholecystectomies were performed in this group of patients. 
We analyzed all patients with a diagnosis of cirrhosis that was based on clinical criteria, laboratory tests, and intraoperative macroscopic examinations of the liver. All results were confirmed by a pathological study.

All patients underwent a programmed LC once the diagnosis of symptomatic cholelithiasis was established based on clinical symptoms (biliary colic, nausea, vomiting, epigastric pain) and then confirmed by means of abdominal ultrasonography.

The liver function was evaluated using a Child-PughTurcotte (C-P-T) score. Preoperative anesthetic risk was measured using the score established by the American Society of Anesthesiologists (ASA).

Before the surgical procedure all patients gave their informed consent. All patients received general anesthesia, and the laparoscopic procedure was similar to that performed for non-cirrhotic patients: $\mathrm{CO}_{2}$ was used for the pneumoperitoneum, and we employed three trocars ( 2 of $10 \mathrm{~mm}$ and 1 of $5 \mathrm{~mm}$ in diameter) following the technique described by Dubois.

Liver biopsies were obtained during surgery from all but two patients in order to confirm the diagnosis and etiology of their cirrhosis. Results were positive in all patients. The remaining two patients had been diagnosed with cirrhosis before surgery by means of a percutaneous biopsy.

We analyzed the conversion rate, duration of surgical procedure, incidence of intraoperative complications (liver bed bleeding, necessity of transfusion, conversion), postoperative complications (ascites, pleural effusion, worsening of C-P-T classification, umbilical hernia), and mean hospital stay.

Patients were followed up for a period of one year after surgery.

\section{RESULTS}

Eight of the 14 patients included in the present study $(57.14 \%)$ were women and $6(42.85 \%)$ were men, with a mean age of 60 yrs. In $71.42 \%$ of cases (10 patients) the origin of cirrhosis was alcoholic, whereas in the remaining $28.57 \%$ (4 patients) it had a viral cause (B and C).

Eight patients (57.14\%) had Child's Class B cirrhosis, and 6 patients $(42.85 \%)$ had Class A cirrhosis. None of the patients in our series had Child's Class C cirrhosis, as no case of cholecystectomy was attended in our hospital during the period of study.

Two of the patients $(14.28 \%)$ presented with portal hypertension $(\mathrm{PH})$, and one of them was diagnosed before surgery because of a DH episode induced by esophageal varices. The other patient was diagnosed during surgery, when we observed a significant increase in splanchnic vascularization.

The ASA score of patients was as follows: 12 patients $(85.71 \%)$ were classified as ASA class II and 2 patients $(14.28 \%)$ as ASA class III. No inhalant anesthetic sub- stances were employed, as they are associated with the development of hepatitis, fulminant hepatic necrosis, and reduced hepatic flow.

None of the patients under study had a previous history of abdominal surgery. And only one patient (7.14\%) had stage-B HIV infection.

The average duration of the surgical procedure was 77 min. Two of the patients (14.28\%) suffered intraoperative complications in the form of liver bed bleeding and required transfusion (1 bag of blood concentrate) during surgery. Three patients $(21.42 \%)$ presented with postoperative complications: $2(14.28 \%)$ suffered from ascites leading to a worsening of the CPT score in one of them, and the third one presented with thoracic pain of ischemic origin.

No conversion form LC to OC occurred, and there was no perioperative mortality.

Mean hospital stay was 3 days. One year after surgery all patients were still receiving follow-up in the Department of Digestive Diseases due to the asymptomatic cirrhosis derived from the surgical procedure. None of the 14 patients required a second surgical intervention.

\section{DISCUSSION}

Cirrhosis has a high prevalence among the Spanish population. It is characterized by a chronic and irreversible alteration of the hepatic parenchyma in combination with fibrosis, which causes a nodule-forming progressive destruction and subsequent regeneration of liver cells. The resulting hepatic dysfunction increases morbidity and mortality rates among these patients by up to $50 \%$ at 5 years after initial diagnosis. Nevertheless, this percentage may vary depending on the etiology of cirrhosis (1).

Cirrhosis is also a factor predisposing to other pathologies that may require surgery (biliary lithiasis, hernias, digestive hemorrhage). The incidence of lithiasis among cirrhotic patients is twice that seen in the general population because of several factors, such as the presence of hypersplenism, elevated estrogen levels, and increased intravascular hemolysis in association with reduced vesicular motility and difficult emptying, which increases the risk of lithogenesis (2-4).

During the 1980s cholecystectomy in cirrhotic patients was associated with a high mortality rate -around $7.27 \%$ - due to blood loss, postoperative deterioration of hepatic functioning, and the presence of sepsis in many of these patients $(3,4)$.

All patients included in the present study had compensated Child's Class A and Class B cirrhosis. In most of the patients, with the exception of two, cirrhosis was diagnosed during surgery through a gross examination of the liver, which was subsequently confirmed by means of intraoperative biopsies and laboratory tests. 
Following the surgical procedure, all patients had deterioration of their liver function, hypoalbuminemia, and coagulation disturbances, which did not have clinical consequences except for two patients (14.28\%) who had a worsening of their Child's Class and developed ascites.

The first report on the use of laparoscopic cholecystectomy in patients with liver cirrhosis was published by Yerdel et al. in 1993. This report proved that LC was a safe procedure, with a short duration and requiring reduced hospital stay $(5,6)$. These positive data were confirmed by subsequent studies, which showed that cirrhotic patients had a better tolerance for LC than for OC, since LC required a smaller incision and therefore reduced postoperative discomfort, the risk of infiltration of ascitic fluid in the wound, and the occurrence of further complications related to the surgical wound, such as infection, dehiscence, and postlaparoscopic hernia.

All these data have also been confirmed by our study, as none of our patients showed complications related to the surgical wound in the period of 1 year of follow-up after surgery.

The above-mentioned reports also established that LC caused less bleeding (6-8). Blood loss during the surgical procedure is one of the most serious perioperative complications when dealing with cirrhotic patients, and it usually occurs in $57 \%$ of patients undergoing laparotomy (8).

This percentage is reduced in the case of laparoscopy thanks to the meticulous handling of this technique, magnification of the operative field, and the specific effects of barohemostasis as determined by pneumoperitoneum (4,8-11).

Hemorrhage in the bladder bed is mainly caused by venous bleeding, which due to its lower pressure may be easily controlled by the positive pressure exerted by the pneumoperitoneum. Two of the patients included in our sample (14.28\%) developed intraoperative complications in the form of liver-bed bleeding, and required blood transfusion.

In the case of patients with portal hypertension, characterized by hypertension at the venous system, many authors advocate for the use of subtotal (supracystic) cholecystectomy in order to avoid intraoperative bleeding (4,12-14).

In our study this technique was not employed, as only two patients showed intraoperative signs of portal hypertension but of no previous clinical significance. Consequently, we decided to perform the standard procedure.

Another advantage of laparoscopy over laparotomy is a lower rate of postoperative adhesions due to the smaller incision it requires, which also favors potential hepatic transplantation in the future $(5,6,12,13,15)$.

Morino et al. have also described the advantages the use of LC in patients with viral cirrhosis has for the staff, as the risk of contamination decreases from 9 to $4.7 \%$ (56 ). In our milieu viral hepatitis is the second cause of cirrhosis and one of the most common causes of contamina- tion of healthcare professionals $-28.57 \%$ of our patients had viral cirrhosis, which in one of them was associated with AIDS. None of the surgical procedures resulted in staff infection.

For patients with Child's Class C cirrhosis, we must carefully evaluate the benefits and risks of all surgical procedures, particularly of cholecystectomy, as the mortality rate associated with both open and laparoscopic cholecystectomy is extremely high, around $27 \%$ according to a report by Block et al. (7).

So far, there is not enough experience in the use of LC for such patients -only two cases were reported in 2003 (8), another case has been reported by Yerdel, and a fourth case was described by Lacy. Currently, the technique to be employed in this type of patients will depend on the surgeon's experience and choice. In our study we included no patients with Child's Class $\mathrm{C}$ cirrhosis, since no LC had been performed in this type of patients in our hospital during the study period.

We may conclude that cholecystectomy is a surgical procedure posing a high risk for cirrhotic patients. Therefore its indication must be carefully considered, and each case must be individually studied.

From our data we observe that LC is a safe and effective alternative in the treatment of symptomatic cholelithiasis for patients with well-compensated Child's Class A and Class B cirrhosis. However, the use and prevalence of this technique in our hospital has been scarce $-30 \%$ (only 14 patients undergoing laparoscopic cholecystectomy vs. 32 undergoing laparotomic cholecystectomy)- during the last 8 years.

\section{REFERENCES}

1. Podolsky DK, Isselbacher KJ. Hepatopatía alcohólica y cirrosis. En: Principios de Medicina Interna. Harrison, 1994. p. 1705-18.

2. Del Olmo JA, Flor-Lorente B, Flor-Civera B, Rodríguez F, Serra MA. Risk facts for nonhepatic surgery in patients with cirrosis. World $\mathrm{J}$ Surg 2003; 10: 268-76.

3. Acalovschi M, Blandea D. Risk factors for symptomatic gallstones in patients with liver cirrhosis: a case-control study. Am J Gastoenter 2003; 98: 1856-60.

4. Acalovschi M, Dumitrascu DL, Nicoara CD. Gallbladder contractility in liver cirrosis: comparative study in patients with and without gallbladder stones. Dig Dis Sci Jan 2004; 49 (1): 17-24.

5. Puggioni A, Wong LN. A metaanalysis of laparoscopic cholecystectomy in patients with cirrhosis. J Am Coll Surg 2003; 197 (6): 921-6.

6. Poggio J1, Rowland CM, Gores GL, et al. A comparison of laparoscopic and open cholecystectomy in patients with compensated cirrhosis and symptomatic gallstone disease. Surgery 2000; 127: 405-11.

7. Yerdel MA, Tsuge H, Mimura H, Sakagami K, Mori M, Orita K. Laparoscopic cholecystectomy in cirrhotic patients: expanding indications. Surg Laparoscoc Endosc 1993; 3: 180-3.

8. Lacy AM, Balaguer C, Andrade E, García-Valdecasas JC, Grande L, Fuster J, et al. Laparoscopic cholecystectomy in cirrhotic patients: indication or contradiction. Surg Endosc 1995; 9: 407-8.

9. Cucinotta E, Lazzara S, Melita G. Laparoscopic cholecystectomy in cirrhotic patients. Surg Endosc 2003; 17 (12): 1958-60.

10. Tuech JJ, Patrick M, Regenet N, Rouge C, Bergamaschi R, et al. Laparoscopic cholecystectomy in cirrotic patients. Surg Laparosc Endosc Percutan Tech 2002; 12 (4): 227-31. 
11. Yeh CN, Chen MF, Jan YY. Laparoscopic cholecystectomy in 226 cirrhotic patients. Experience of a single center in Taiwan. Surg Endosc 2002; 16 (11): 1583-7.

12. Fernandes NF, Schwesinger WH, Hilsenbeck SG, Gross GW, Bay MK, Sirinek KR, et al. Laparoscopic cholecystectomy and cirrhosis: a case-control study of outcomes. Liver Transpl 2000; 6 (3): 340-4.

13. Urban L, Eason GA, ReMine S, Bogard B, Magisano $\mathrm{J}$, Raj P, et al. Laparoscopic cholecystectomy in atients with early cirrhosis. Curr Surg. 2001; 58 (3) 312-5.

14. JiW LiLT, ChenXR LiJS. Application of laparoscopic cholecystectomy in patients with cirrhotic portal hypertension. Hepatobiliary Pancreat Dis Int 2004; 3 (2): 270-4.

15. Morino, M, Cavuoti, G. Laparoscopic cholecystectomy in cirrosis: contraindicacion or privileged indication? Laparosc Endosc Percutan Tech 2000; 10: 360-3. 